\title{
Haemorrhage Secondary to Cerebral Aneurysm in a Senegalese Patient with Systemic Lupus Erythematosus: A Case Report
}

\author{
Baïdy Sy Kane, Maïmouna Sow, Boundia Djiba, Awa Cheikh Ndao, Mohamed Dieng, \\ Souhaibou Ndongo, Abdoulaye Pouye \\ Department of Internal Medicine, Le Dantec Teaching Hospital, Cheikh Anta DIOP University, Dakar, Senegal \\ Email: baidykane@gmail.com, mouna89sow@hotmail.com, boundiadjiba@yahoo.fr, ndaoawacheikh@gmail.com, \\ docmdieng@gmail.com, sndongo_medinterne@gmail.com, docpouye@yahoo.fr
}

How to cite this paper: Kane, B.S., Sow, M., Djiba, B., Ndao, A.C., Dieng, M., Ndongo, S. and Pouye, A. (2019) Haemorrhage Secondary to Cerebral Aneurysm in a Senegalese Patient with Systemic Lupus Erythematosus: A Case Report. Health, 11, 1625-1630. https://doi.org/10.4236/health.2019.1112123

Received: December 8, 2019

Accepted: December 24, 2019

Published: December 27, 2019

Copyright $\odot 2019$ by author(s) and Scientific Research Publishing Inc. This work is licensed under the Creative Commons Attribution International License (CC BY 4.0).

http://creativecommons.org/licenses/by/4.0/

\begin{abstract}
Introduction: Hemorrhage by ruptured cerebral aneurysm is a rare and severe complication of systemic lupus erythematosus (SLE). We reported a case of this complication. Observation: A 24-year-old woman black Senegalese patient was followed in our department since for a systemic lupus with cutaneous and articular involvement and class III and V Lupus nephritis. She was readmitted for acute headache and early postprandial vomiting. The examination showed a meningeal syndrome, a subacute lupus eruption in the trunk, panniculitis and fever. The cerebral computer tomography showed spontaneous haemorrhage from saccular aneurysm. She was managed by immediate aneurysm clipping and medical treatment including bolus of methylprednisolone and immunosuppressive therapy was maintained. The outcome was favourable, but there was neurological damage such as brachial weakness. Conclusion: Hemorrhage by ruptured cerebral aneurysm is a rare and severe complication of SLE. The risk of damage is also significant. Immediate neurosurgical management and aggressive medical treatment may improve the prognosis.
\end{abstract}

\section{Keywords}

Systemic Lupus Erythematosus, Central Nervous System Lupus, Subarachnoid Hemorrhage, Africa South of the Sahara

\section{Introduction}

Systemic Lupus Erythematosus (SLE) is a complex systemic auto-immune disease characterized by a wide spectrum of clinical, laboratory and immunological 
abnormalities and a variable course and outcome [1].

It represents a common systemic autoimmune disease of unknown cause [2] [3] [4] [5].

The central, psychiatric and peripheral neurological manifestations of lupus are among the most severe visceral disorders and are grouped under the general term of "neuro-psychiatric systemic lupus erythematosus" (NPSLE) [6]. In 1999, an expert panel of the American College of Rheumatology (ACR) defined 19 clinical situations that are observed with NPSLE. This classification includes a wide range of syndromes and distinguishes between central and peripheral manifestations of NPSLE (12 in the central nervous system (CNS) and 7 in the peripheral nervous system) [7].

The overall prevalence of these events varies significantly between studies, ranging from $20 \%$ to $97 \%$ [8] [9] [10]. In a recent meta-analysis identifying all of the studies based on the ACR criteria and identifying the most robust from a methodological point of view, the overall prevalence of neuropsychiatric manifestations was estimated at 56\% [11]. Among the NPSLE, there are cerebrovascular accidents which are mostly ischemic and rarely haemorrhagic [12]. Subarachnoid haemorrhages (SAH) secondary to cerebral aneurysms related to SLE are exceptionally reported [13].

We described a case of SAH secondary to ruptured aneurysm in a Senegalese black patient with SLE.

\section{Observation}

A 24-year-old woman black Senegalese patient was followed in our Internal Medicine department since 2012 for a SLE with cutaneous and articular manifestations and class III and V Lupus nephritis. In addition to corticosteroids and synthetic antimalarial drugs, she had received several immunosuppressants (cyclophosphamide, mofetil mycophenolate and azathioprine as maintenance therapy).

SLE was associated with an anti-phospholipid syndrome discovered during a pulmonary embolism that justified prolonged anticoagulation with Acenocoumarin at $4 \mathrm{mg} /$ day.

In her previous follow-up, she was also admitted in our department in 2014 for a suspected NPSLE. The brain magnetic resonance imaging (MRI) was performed in this hospitalization and was normal.

She was readmitted in August 2016 for the treatment of a meningeal syndrome.

The interrogation found acute headache resistant to usual analgesics, partially calmed by early post prandial vomiting.

On admission, the temperature was $39^{\circ} \mathrm{C}$, the heart rate 102 beats per minute, the respiratory rate at 20 cycles per minute and the blood brachial pressure at $110 / 80 \mathrm{~mm}$ hg.

Consciousness was clear with a Glasgow score of 15/15. The examination showed a meningeal syndrome, without neurological weakness. The remainder of the examination revealed subacute cutaneous lupus with an erythematous, annular 
lesion in the trunk and lupus panniculitis lesions.

The blood cells count showed leukopenia at 3630 with lymphopenia at 182 elements $/ \mathrm{mm}^{3}$ and neutrophil polynuclear cells at 3449 elements $/ \mathrm{mm}^{3}$. The haemoglobin level was $6 \mathrm{~g} / \mathrm{dl}$ and platelets count at $302.000 / \mathrm{mm}^{3}$. The erythrocyte sedimentation rate was $105 \mathrm{~mm}$ at the first hour and the C-reactive protein at 24 $\mathrm{mg} / \mathrm{l}$. The International Normalized Ratio was 1.65 (under $4 \mathrm{mg}$ of Acenocoumarin). The remainder of the biological assessment included blood urea at 0.16 $\mathrm{mg} / \mathrm{l}$, creatinine at $05.4 \mathrm{mg} / \mathrm{l}$, serum protein at $69.6 \mathrm{~g} / \mathrm{l}$ and albuminemia at 33.8 g/l. SLE was active with a clinical Systemic Lupus Erythematosus Disease Activity Index (cSLEDAI) at 19.

The infection rate (cytobacteriological examination of urine, cerebrospinal fluid, blood cultures, thick drop) was negative.

The cerebral computer tomography found spontaneous right internal front o-temporal hyperdensity with mass effect and almost complete collapse of the frontal horn of the homolateral ventricle surrounded by oedema and discrete sub-falcorian engagement. After the injection of contrast, a saccular aneurysm of about $13 \times 10 \mathrm{~mm}$ was observed along the path of the middle cerebral artery with a neck at $02 \mathrm{~mm}$ (Figure 1).

The patient was transferred to the Neurosurgical department. An immediate endovascular treatment was performed by surgicalaneurysm clipping. She received also methylprednisolone. Hydroxychloroquine (400 mg) and azathioprine (100 mg, immunosuppressive therapy) were also maintained.

The short-term course was marked by the development of an incomplete left hemiplegia without intracranial hypertension syndrome or meningeal syndrome.

After three years follow up, there is a neurological sequalae such as brachial paralysis without lupus flairs or relapse of the neurologic events.

\section{Discussion}

We reported a case of SAH during SLE.

This complication has been rarely reported in the international literature. Mimori et al., identified 10 cases in a population of 258 patients, over a 20 -year period, representing a prevalence of $3.9 \%$ [12]. The incidence was also estimated at $0.1 \%$ [13].

A meta-analysis of stroke in SLE, using population-based cohort studies by Holmqvist et al., showed that individuals with SLE have a threefold higher risk of intracerebral hemorrhage, and an almost fourfold increase in SAH risk [14] [15].

The formation of aneurysm secondary to SLE has been related to inflammation of the vascular wall associated with fibroid necrosis [13].

This complication would occur as well as our observation, in patients under 50 years of age and with active SLE. Our patient had active disease and LN. In the report of Mimori et al., 07 patients (total of 10 patients) had LN and the disease was active in half of the cases [12]. Similar data, about association with LN have been also reported in the literature [16] [17]. This could be related to the severity of the disease. 


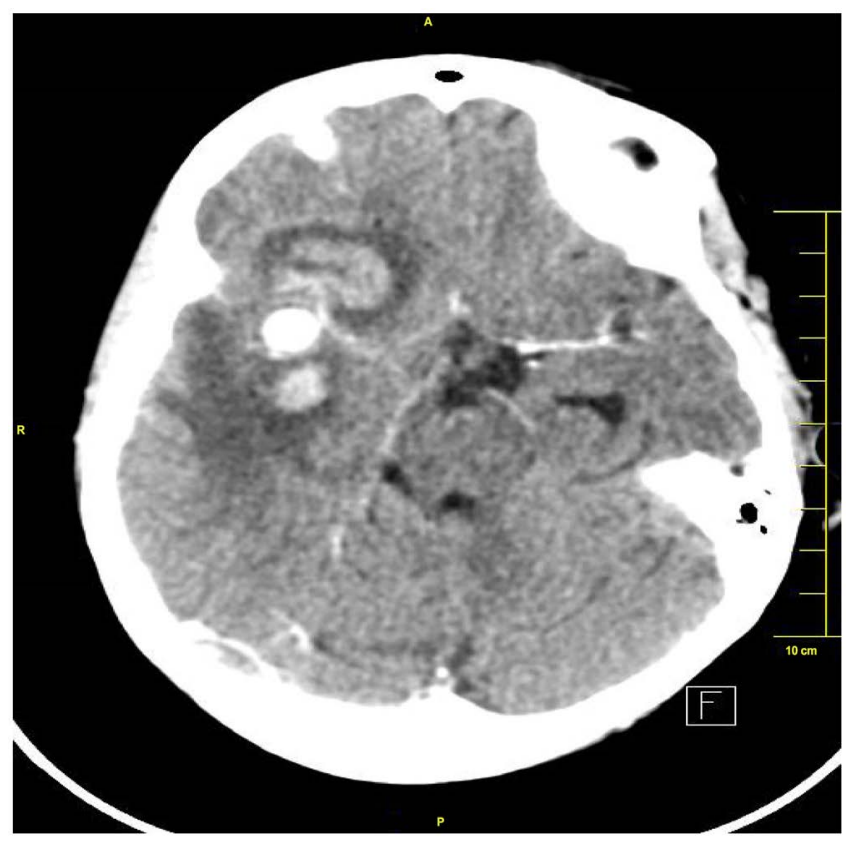

Figure 1. Axial computer tomography. Haemorrhagic stroke by aneurysm rupture in our patient.

The diagnosis was made in our observation by Computer tomography angiography. It identified one saccular aneurysm, noted in half of the cases in the literature [13]. The multiple and fusiform character would be more associated with SLE cerebral vasculitis [13].

Emergency neurosurgical treatment is based on the placement of a clip or endovascular coil embolization. The prevention of vasospasm and the management of hydrocephalus would be conducted [13]. Medical treatment is not codified, as other subtype of NPSLE could be based on methylprednisolone and cyclophosphamide.

Mortality rate associated with aneurysm rupture during SLE is high, estimated at $60 \%$ [13]. SAH accompanied by active SLEwas associated with a poor prognosis, the five patients of Mamori's study with active SLE had died [12]. Immediate neurosurgical management and the aggressive medical treatment (methylprednisolone bolus and immunosuppressants) could improve the prognosis of SAH with active SLE, similarly to our case.

The main limitation of our work is its nature which not allow draw definitive conclusion. However, this case report should contribute to a better knowledge rare complication of SLE and his management.

\section{Conclusion}

Hemorrhage by ruptured cerebral aneurysm is a rare and severe complication of SLE. The risk of damage is also significant. It may be associated with an age less than 50 years-old, disease activity and lupus nephritis. Immediate neurosurgical management and aggressive medical treatment may improve the prognosis. 


\section{Consent for Publication}

Written informed consent for publication was obtained from the patients.

\section{Conflicts of Interest}

The authors declare no conflicts of interest regarding the publication of this paper.

\section{References}

[1] Felten, R., Dervoric, E., Chasset, F., Gottenberg, J.E., Sibilia, J., Scher, F. and Arnaud, L. (2018) The 2018 Pipeline of Targeted Therapies under Clinical Development for Systemic Lupus Erythematosus: A Systematic Review of Trials. Autoimmunity Reviews, 17, 781-790. https://doi.org/10.1016/j.autrev.2018.02.011

[2] Nossent, J.C. and Swaak, A.J. (1991) Prevalence and Significance of Haematological Abnormalities in Patients with Systemic Lupus Erythematosus. The Quarterly Journal of Medicine, 80, 605-612.

[3] Keeling, D.M. and Isenberg, D.A. (1993) Haematological Manifestations of Systemic Lupus Erythematosus. Blood Reviews, 7, 199-207. https://doi.org/10.1016/0268-960X(93)90006-P

[4] Van Dam, A.P. (1991) Diagnosis and Pathogenesis of CNS Lupus. Rheumatology International, 11, 1-11. https://doi.org/10.1007/BF00290244

[5] Carbotte, R.M., Denburg, S.D. and Denburg, J.A. (1986) Prevalence of Cognitive Impairment in Systemic Lupus Erythematosus. The Journal of Nervous and Mental Disease, 174, 357-364. https://doi.org/10.1097/00005053-198606000-00008

[6] Schwartz, N., Stock, A.D. and Putterman, C. (2019) Neuropsychiatric Lupus: New Mechanistic Insights and Future Treatment Directions. Nature Reviews Rheumatology, 15, 137-152. https://doi.org/10.1038/s41584-018-0156-8

[7] (1999) The American College of Rheumatology Nomenclature and Case Definitions for Neuropsychiatric Lupus Syndromes. Arthritis \& Rheumatology, 42, 599-608. https://doi.org/10.1002/1529-0131(199904)42:4<599::AID-ANR2>3.0.CO;2-F

[8] Lefèvre, G., Zéphir, H., Warembourg, F., Michelin, E., et al. (2012) Neuropsychiatric Systemic Lupus Erythematosus (1st Part). Cases Definitions and Diagnosis and Treatment of Central Nervous System and Psychiatric Manifestations of Systemic Lupus Erythematosus. La Revue de Médecine Interne, 33, 491-502. https://doi.org/10.1016/j.revmed.2012.03.356

[9] Ainiala, H., Loukkola, J., Peltola, J., Korpela, M. and Hietaharju, A. (2001) The Prevalence of Neuropsychiatric Syndromes in Systemic Lupus Erythematosus. Neurology, 57, 496-500. https://doi.org/10.1212/WNL.57.3.496

[10] Bertsias, G.K. and Boumpas, D.T. (2010) Pathogenesis, Diagnosis and Management of Neuropsychiatric SLE Manifestations. Nature Reviews Rheumatology, 6, 358-367. https://doi.org/10.1038/nrrheum.2010.62

[11] Brey, R.L., Holliday, S.L., Saklad, A.R., Navarrete, M.G., Hermosillo-Romo, D., Stallworth, C.L., et al. (2002) Neuropsychiatric Syndromes in Lupus: Prevalence Using Standardized Definitions. Neurology, 58, 1214-1220.

https://doi.org/10.1212/WNL.58.8.1214

[12] Mimori, A., Suzuki, T., Hashimoto, M., Nara, H., Yoshio, T., Masuyama, J.-I., et al. (2000) Subarachnoid Hemorrhage and Systemic Lupus Erythematosus. Lupus, 9, 521-526. https://doi.org/10.1177/096120330000900708 
[13] Brah, S., Thomas, G., Chapon, F., Franques, J., Jourde, N., Harlé, J.R., et al. (2012) Subarachnoid Hemorrhages from Ruptured Aneurysms as the Presenting Feature of Lupus Cerebral Vasculitis. La Revue de Médecine Interne, 33, e10-e13. https://doi.org/10.1016/j.revmed.2011.02.006

[14] Holmqvist, M., Simard, J.F., Asplund, K. and Arkema, E.V. (2015) Stroke in Systemic Lupus Erythematosus: A Meta-Analysis of Population-Based Cohort Studies. RMD Open, 1, e000168. https://doi.org/10.1136/rmdopen-2015-000168

[15] Ciril, K., Andrew, C., David, C., Nooshin, K.B., Sein, Y.S. and Bruce, F.G. (2018) Systemic Lupus Erythematosus and Antineutrophilic Cytoplasmic Antibody-Associated Vasculitis Overlap Syndrome Complicated by Subarachnoid Hemorrhage: Case-Based Review. Rheumatology International, 38, 2329-2335. https://doi.org/10.1007/s00296-018-4169-Z

[16] Nakai, Y., Hyodo, A., Yanaka, K., Akutsu, H. and Nose, T. (2000) Distal Superior Cerebellar Artery Aneurysm in a Patient with Systemic Lupus Erythematosus. Surgical Neurology, 54, 73-76. https://doi.org/10.1016/S0090-3019(00)00261-5

[17] Takemoto, K., Hatano, T., Abekura, Y., Takahashi, J.C. and Miyamoto, S. (2013) Successful Flow Reduction Treatment for a Middle Cerebral Artery Aneurysm in a Patient with Systemic Lupus Erythematosus. Neurologia Medico-Chirurgica, 53, 192-195. https://doi.org/10.2176/nmc.53.192 